Article

\title{
Dynamic Changes of Fruit Shape Traits and Its Correlation to the Morphology of Other Organs in Cucumber
}

\author{
Mingming Cui ${ }^{\dagger}$, Yue Yang ${ }^{\dagger}$, Zhihui Cheng and Huanwen Meng * \\ College of Horticulture, Northwest A\&F University, Yangling, Xianyang 712100, China; \\ cuimm@nwafu.edu.cn (M.C.); sailor_yy5683@163.com (Y.Y.); chengzh@nwsuaf.edu.cn (Z.C.) \\ * Correspondence: menghw2005@nwsuaf.edu.cn \\ + These authors have contributed equally to this study.
}

Received: 30 June 2020; Accepted: 28 July 2020; Published: 31 July 2020

\begin{abstract}
Fruit shape and size are important assessment parameters to evaluate and classify accessions in cucumber breeding. Clarifying the relations of the shape and size of commodity fruits or mature fruits to that of other organs, like seed, leaf, flower and so on, is significant for multitype breeding of cucumber fruit. It can help breeders to predict the shape and size of a cucumber fruit in the early selection and shorten the breeding cycle. In this study, the dynamic morphological observation of fruit length (FL), fruit diameter (FD) and the fruit shape index (FSI) of developing cucumber fruits in different types and the correlation of fruit shape indexes between fruit and other organs were investigated. The results showed that 28 cucumber accessions with different shape and size were classified into four types, namely long fruits, medium long fruits, medium fruits and short fruits. The comparative analysis of FL and FD among different types and different development stages of cucumber fruits showed that the development of FL and FD were both consistent with the model "S" curve, slow-fast-slow, and there existed strong positive correlation between their growth. During fruit development, the shape index was highly positively correlated among ovary, commodity fruit and mature fruit. Besides, both the length and width of cotyledon, true leaf and female flower petal were positively correlated to the FL of commodity fruit. The length and width of seed, length of ovary carpopodium and pedicel length were also positively correlated to the FD of commodity fruit. For seed, cotyledon and true leaf, no correlation was found between their shape index and their FSI.
\end{abstract}

Keywords: cucumber; fruit shape and size; FSI; during fruit developing; correlation to other organs

\section{Introduction}

Cucumber (Cucumis sativus L.) is an economic and consumption important cucurbits crop worldwide [1]. As a fruity vegetable, its external qualities play a critical role in the market [2]. Fruit shape and size, as direct appearance quality traits, are main assessment parameters in the evaluation and classification of cucumber accessions [3]. Moreover, in consumption, different sizes of cucumber fruit are preferred in different zones [4]. For example, longer cucumbers are produced and consumed in China and Europe, while shorter cucumbers are more popular in the Mediterranean. Therefore, multiple breeding for fruit shape and size is increasing importantly and clarifying the development regulation of fruit shape and size can provide basic theoretical knowledge for breeding.

Generally, the development of cucumber fruit goes through four stages $[5,6]$. The first stage is the formation and development of ovary. The second stage is the division stage of cells in the fruit, which has a critical influence on the number of cells and shape and size of fruit. The third stage is the expansion stage of cells in the fruit, in which the seed starts to develop and the flesh cell expands, 
which can greatly affect fruit shape. The last stage is the ripening stage of the fruit, in which the shape and size of the fruit has formed and will then show little change. During this developing process, both fruit length (FL) and fruit diameter (FD) are dynamically changing, which may not only determine the fruit shape index (FSI, ratio of FL to FD) of a commodity fruit (appearance quality of fruit shape and size), but also affect cucumber production [7-9]. In general, in the growth and development of cucumber, the tissue cells in pericarp and placenta increase firstly; then all the cells gradually expand. The cell division and expansion phase of cucumber usually ends at two weeks after flowering [10]. However, previous studies showed the specific development process of various cucumber accessions appeared different due to various varieties and cultural conditions [11].

Since recent decades, some studies have been conducted on the growth characteristics of cucumber fruits. Ding reported that in cucumber "11CP-4", FL and FD increased fast at first then slowed down before commodity maturity, 14 days after anthesis (DAA) [12]. In cucumber "Jin Chun 5", it was revealed that both the FL and FD developed in the model of an "S" curve during 0-10 DAA: Slowly before flowering, fast after anthesis (2-4 DAA) and slowly again after 6-8 DAA [13]. In cucumber "9930", FL and FD showed similar growth curves, increasing almost exponentially between 3 DAA and 16 DAA, especially on 3-5 DAA [14]. Another similar study was conducted with four cultivars, and indicated that the rapid development period of FL was during 7-13 DAA and that of FD was during 5-7 DAA and 13-15 DAA in different accessions [10]. The difference could be found among these results, and it may due to the limitation that few cucumber accessions were used. Moreover, previous studies only focused on the change of fruit shape during commodity fruit development, and little systematic study was aimed at the fruit shape and size change during the whole development process (from flowering to fully mature fruit) of a fruit in multitype fruit cucumber accessions. Therefore, in this work, a larger population of 28 cucumber accessions with different shape and size were used to accumulate knowledge of cucumber fruit shape change during fruit dynamic development process.

Agronomic traits are the result of a series of complex biochemical reactions in plants. Because different traits may be regulated by the same gene or have the same biochemical reaction link, certain correlations are often shown between different traits. Wang et al. conducted a study on the correlation between the size of strawberry fruit and 12 other agriculture characters [15]. Results showed that the crown diameter, leaf area, stem thickness, plant height, petals area, stem length and calyx area were significantly positively correlated with the size of the fruit, and leaf shape index and fruit shape index, leaf color and fruit color were also closely related. A study on morphological analysis of cucumber was also conducted and results showed that there was a moderate positive correlation between the length of fruit and the thickness of flesh and the length of stem [16]. However, studies on the correlations among cucumber traits usually focus on sensory quality and yield; few studies focus on the correlation of fruit shape. Therefore, in this study, 28 cucumber accessions with bar fruits and one cucumber accession with round fruit were employed to investigate the correlations between the shape index of a commodity fruit or a fully mature fruit and the shape of other organs in cucumber.

\section{Materials and Methods}

\subsection{Plant Materials and Their Cultivation}

In total, 28 typical cucumber inbred lines with different fruit shapes and sizes were employed in this study, namely: G4, 74-18, 3511, QK, 58-621, RL-10, JS-11, J431, XY-4, 39-2, 7-2, 4-2, FCH-5, MCH-4, 39-1, 4, 2016-4-1, 8, 1, 2016-4-2, 29, 3-1, 20, 22-1, 24-2, 23, Q14-1 and 16-1. The origins and genotypes are given in Table S4. All cucumber accessions were provided by the Cucumber Research Group of College of Horticulture, Northwest A\&F University, Yangling, Shaanxi province, China.

Seeds for seedling and fruit experiment were treated with hot water at $55^{\circ} \mathrm{C}$ for $15 \mathrm{~min}$, followed by soaking in water at $30^{\circ} \mathrm{C}$ for $6 \mathrm{~h}$, and then pre-germinated in an incubator under $28^{\circ} \mathrm{C}$ and humid conditions. Then the germinated seeds were sowed in a 72 hole plug tray and put in a growth chamber with temperature and light conditions as following: $12 \mathrm{~h}$ of lighting at $30 \mathrm{klx}, 25^{\circ} \mathrm{C} / 12 \mathrm{~h}$ darkness, 
$18^{\circ} \mathrm{C}$. The seedlings were transplanted at about the three true leaf stage in spring and grown in summer in plastic greenhouses ( $50 \mathrm{~m}$ long and $8 \mathrm{~m}$ wide) under natural sunlight conditions at the Horticultural Research Station, Northwest A\&F University (E $\left.108^{\circ} 08^{\prime}, \mathrm{N}_{34^{\circ}}^{\circ} 2^{\prime}\right)$.

\subsection{Phenotypic Data Collection}

Ten seeds of each accession were randomly sampled, and the length and the width of each seed were measured with a vernier caliper. The measurement was triplicated for all seeds.

All the ten seeds measured and another twenty seeds of each accession were sowed, among which ten seedlings were randomly sampled (when cotyledons and the first true leaf were fully stretched) to measure the length and width of cotyledon and the first true leaf blade, and the petiole length of the first true leaf with a ruler or a vernier caliper. The measurement was triplicated for all seedlings. Then, all these selected plants were transplanted and used for the next measurement.

In a plastic greenhouse, one healthy female flower between the 8th and 12th nodes of each cucumber plant (ten in total) of each accession was randomly selected and labeled at flowering stage. The length and width of a fully opened female flower petal and the length and width of the ovary and ovary carpopodium were measured with ruler or vernier caliper. Then the FL and FD of the labeled young fruit of each accession were carefully measured on a daily basis during 0-8 DAA and at 11, 14, 24,34 DAA. The fruits at 11 DAA were selected to represent commodity fruit and the length and width of the pedicel were also measured with ruler or vernier caliper. The fruits at 34 DAA were measured to represent mature fruit. All the measurements were performed in three replications.

The shape index of all measured organs (seed, cotyledon, true leaf, petal and carpopodium of female flower, fruit and its pedicel) was calculated in accordance with the formula:

$$
\text { Shape index }=\frac{\text { length of organ }}{\text { width of organ }}
$$

\subsection{Data Process}

Microsoft Excel.2019 and IBM SPSS Statistics 23.lnk were used to analyze and plot the data collected.

In cluster classification, systematic cluster analysis was used and 20 indexes were employed: Length and width of seed, cotyledon, 1st leaf, petal, ovary, ovary carpopodium, commodity fruit, mature fruit, carpopodium of commodity fruit, petiole length of cotyledon and that of 1st leaf.

Tukey HSD tests were used for comparison of mean among indexes of different types of cucumber fruit at $p<0.05$. Correlations were calculated with the Pearson test at $p<0.05$.

\section{Results}

\subsection{Clustering Analysis}

Based on data of 20 indexes collected, a cluster analysis was performed and 27 accessions with bar fruits were classified into four types (Figure 1), namely long fruits, medium long fruits, medium fruits and short fruits. The long fruit type contains eleven accessions: 58-621, J431, XY-4, 39-2, G4, 3511, RL-10, 7-2, QK, 29, JS-11; the medium long fruit type contains six accessions:74-18, 42, FCH-5, 39-1, MCH-4, 1; the medium fruit type contains five accessions: 4, 2012-4-1, 2012-4-2, 22-1, 23; and the short fruit type contains five accessions: Q14-1, 24-2, 8, 3-1, 20. The length of the four types of commodity fruits is $373-424 \mathrm{~mm}, 242-299 \mathrm{~mm}, 181-198 \mathrm{~mm}$ and $120-165 \mathrm{~mm}$, respectively. The width of the four types of commodity fruits is $36-43 \mathrm{~mm}, 30-43 \mathrm{~mm}, 36-50 \mathrm{~mm}$ and $42-50 \mathrm{~mm}$ respectively. 


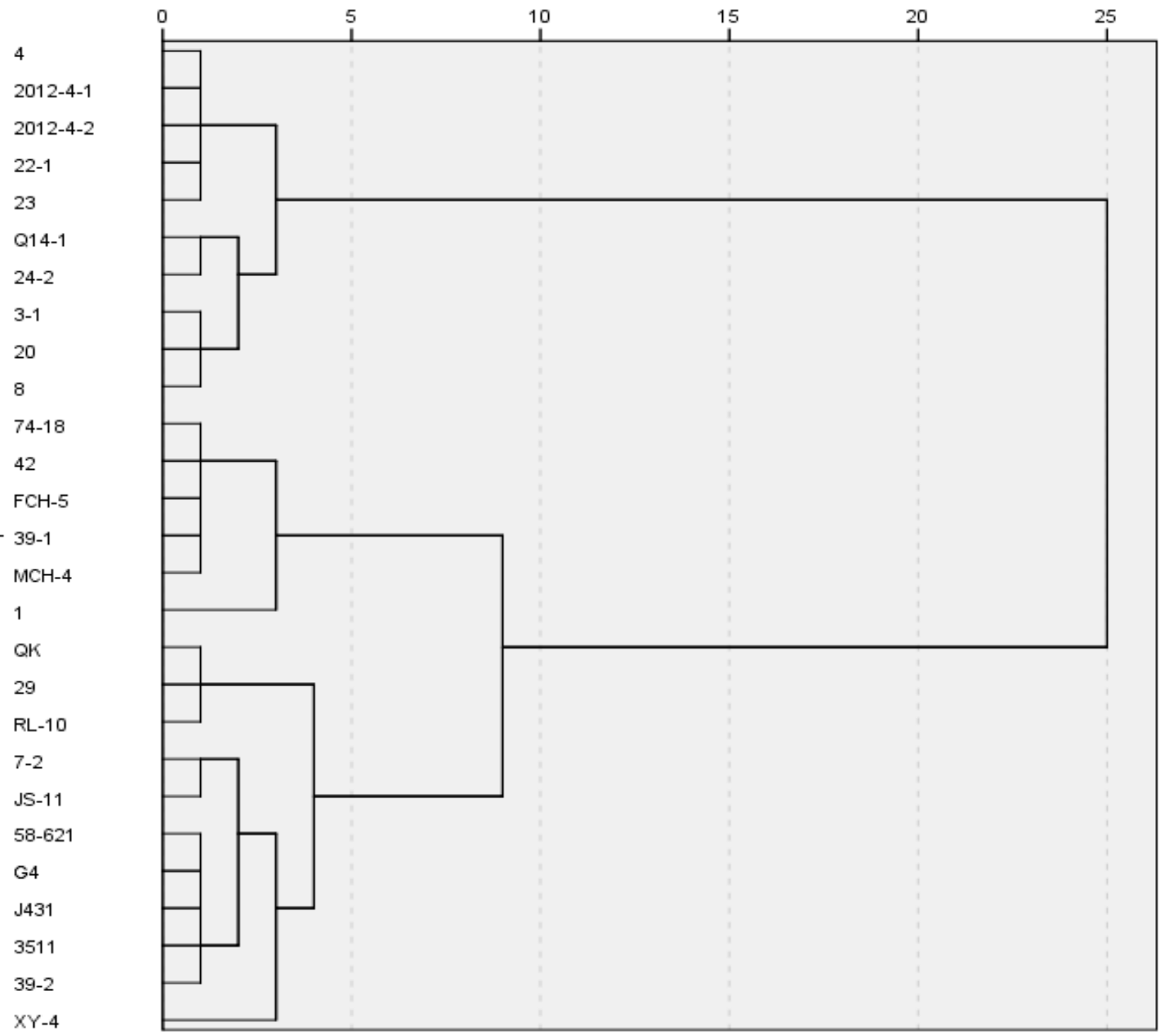

Figure 1. Cluster classification of 27 cucumber accessions based on data of 20 indexes.

\subsection{The Dynamic Change of FSI in Four Types of Cucumber Fruits}

In four types of bar fruits, the change of FSI showed a "Double-S" curve pattern, especially in the long fruits and medium long fruits (Figure 2). For long fruits and medium long fruits, the FSI increased sharply during 0-4 DAA, dropped during 4-6 DAA, increased again during 6-7 DAA and decreased and kept stable after 8 DAA. This fluctuating trend indicated that FL growth started earlier or increased faster than FD at the early stage of fruit development in long fruits and medium long fruits, and then the growth of FD sped up. After the second expansion in FL and FD alternately, the FSI became stable, which indicated that the developing fruits kept a synchronous increasing speed in FL and FD during the middle and late developing stages. For medium fruits and short fruits, the FSI were very stable throughout all the developing stages even at the early developing stage, which indicated that the increase in FL and FD of these two kinds of cucumber fruits almost kept a synchronous and stable developing speed. In cucumber accession "16-1", the FSI was a little bit larger in the early developing stage (0-11 DAA) and then kept stable (Figure S1), which is similar with medium fruits and short fruits. 


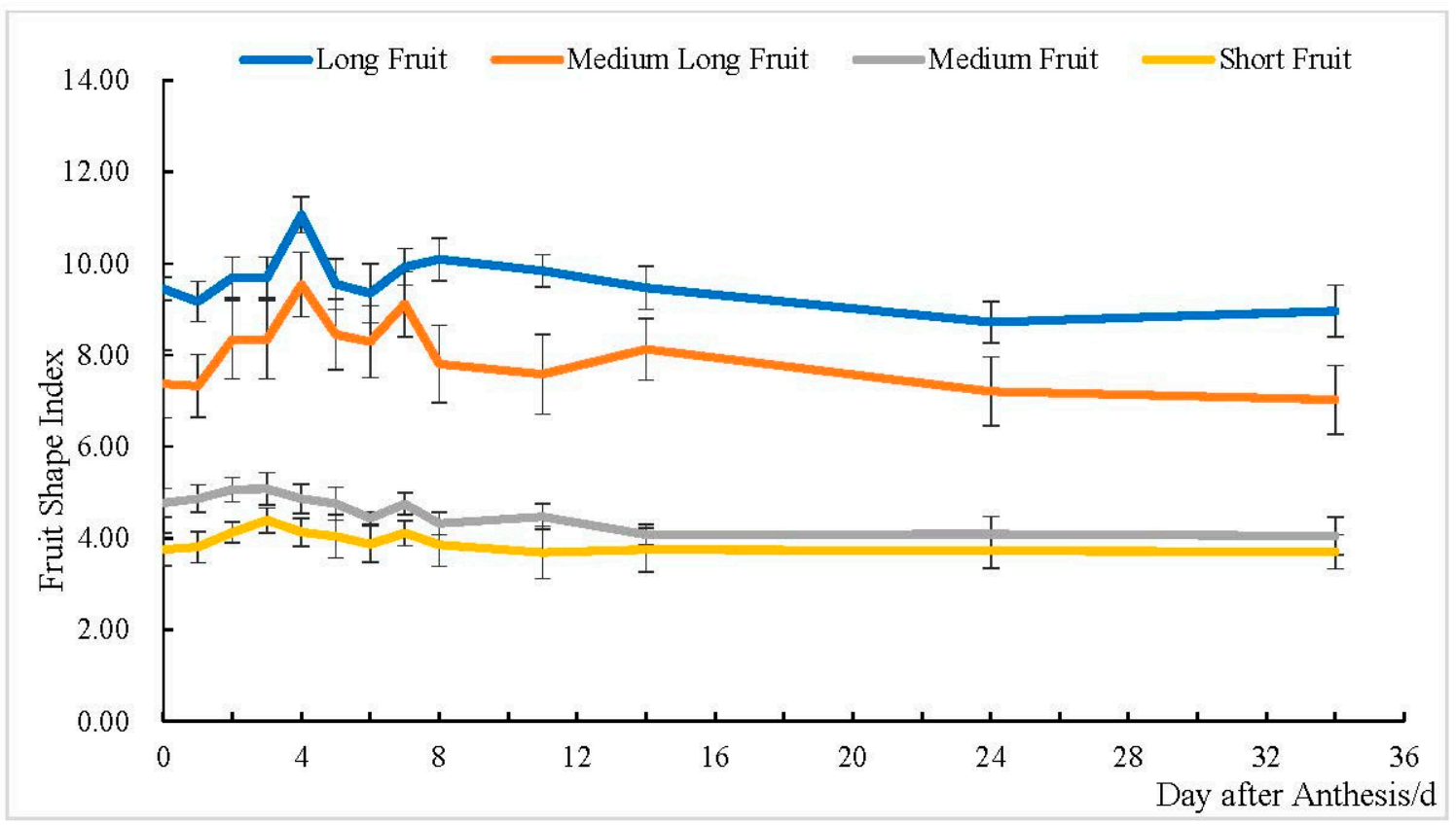

Figure 2. The dynamic changes of fruit shape index in four types of cucumber fruits during the fruit developing process.

\subsection{The Shape Features of Commodity Fruit and Mature Fruit in Cucumber}

For four types of bar fruits, the comparison of FL, FD and FSI between commodity fruits and mature fruits was conducted (Table 1$)$. For FL, significant difference $(p<0.05)$ was found among all types of bar fruits at the commodity fruit stage, but no significant difference was found between long fruits and medium long fruits, nor between medium fruits and short fruits at the mature fruit stage.

Table 1. Fruit shape traits of four types of cucumber fruits different in shape and size.

\begin{tabular}{ccccccc}
\hline \multirow{2}{*}{ Fruit type } & \multicolumn{3}{c}{ Commodity Fruit } & \multicolumn{3}{c}{ Mature Fruit } \\
\cline { 2 - 7 } & FL $(\mathbf{m m})$ & FD $(\mathbf{m m})$ & FSI & FL $\mathbf{( m m})$ & FD $(\mathbf{m m})$ & FSI \\
\hline Long fruit & $388.4 \pm 5.0 \mathrm{a}$ & $40.37 \pm 1.09 \mathrm{a}$ & $9.87 \pm 0.29 \mathrm{a}$ & $516.2 \pm 15.4 \mathrm{a}$ & $58.78 \pm 4.06 \mathrm{a}$ & $9.84 \pm 0.35 \mathrm{a}$ \\
Medium long fruit & $272.1 \pm 5.2 \mathrm{~b}$ & $38.88 \pm 2.13 \mathrm{a}$ & $7.61 \pm 0.35 \mathrm{~b}$ & $432.5 \pm 27.9 \mathrm{a}$ & $64.34 \pm 6.10 \mathrm{a}$ & $7.58 \pm 0.87 \mathrm{a}$ \\
Medium fruit & $193.3 \pm 3.3 \mathrm{c}$ & $43.83 \pm 1.29 \mathrm{~b}$ & $4.50 \pm 0.13 \mathrm{c}$ & $268.6 \pm 26.2 \mathrm{~b}$ & $66.82 \pm 4.61 \mathrm{a}$ & $4.47 \pm 0.28 \mathrm{~b}$ \\
Short fruit & $147.3 \pm 4.4 \mathrm{~d}$ & $42.10 \pm 1.69 \mathrm{~b}$ & $3.70 \pm 0.22 \mathrm{c}$ & $229.1 \pm 17.1 \mathrm{~b}$ & $62.56 \pm 1.77 \mathrm{a}$ & $3.69 \pm 0.57 \mathrm{~b}$ \\
\hline
\end{tabular}

Different letters indicate significant differences between means within columns of four types of cucumber fruits at $p<0.05$ by Tukey HSD test.

For FD, there was no significant difference between long fruits and medium long fruits, and between medium fruits and short fruits, but long fruits and medium long fruits were both significantly thicker than medium fruits and short fruits at the commodity fruit stage. While for mature fruits, no difference was found among all four types of fruits in FD.

For FSI, long fruits appeared to have the highest value and short fruits showed the lowest value. For commodity fruit, there existed significant difference among long fruits, medium long fruits and medium fruits, and the FSI of short fruits was significantly lower than that of long fruits and medium long fruits. For mature fruit, the differences in FSI among all types of cucumber fruits were the same as that in FL.

From Pearson correlation analysis, high positive correlation between FSI and FL $(r=0.852 * *$, $r=0.894^{* *}$, respectively, in commodity fruit and mature fruit; ${ }^{* *}$ correlation is significant at the 0.01 level) and high negative correlation between FSI and FD $\left(r=-0.559 * *, r=-0.562^{* *}\right.$, respectively, in commodity fruit and mature fruit; ${ }^{* *}$ correlation is significant at the 0.01 level) were found. This result 
indicates that FSI is a composite trait and its value is highly influenced by both component traits of FL and FD.

The dynamic observation and comparative analysis of FL and FD were conducted in both sets of four types of bar fruits and round fruits. For bar fruit, the development of FL showed an "S" curve trend-slow (before 3 DAA)-fast (3-15 DAA)-slow (after 15 DAA)-and FD developed following FL with specific positive correlation in different types of fruit. However, the stage of fast increase of FD showed later than that of FL (5-15 DAA) and the variation was a little flatter than FL (Figure 3a-d), indicating the FL developed faster and earlier than the FD.
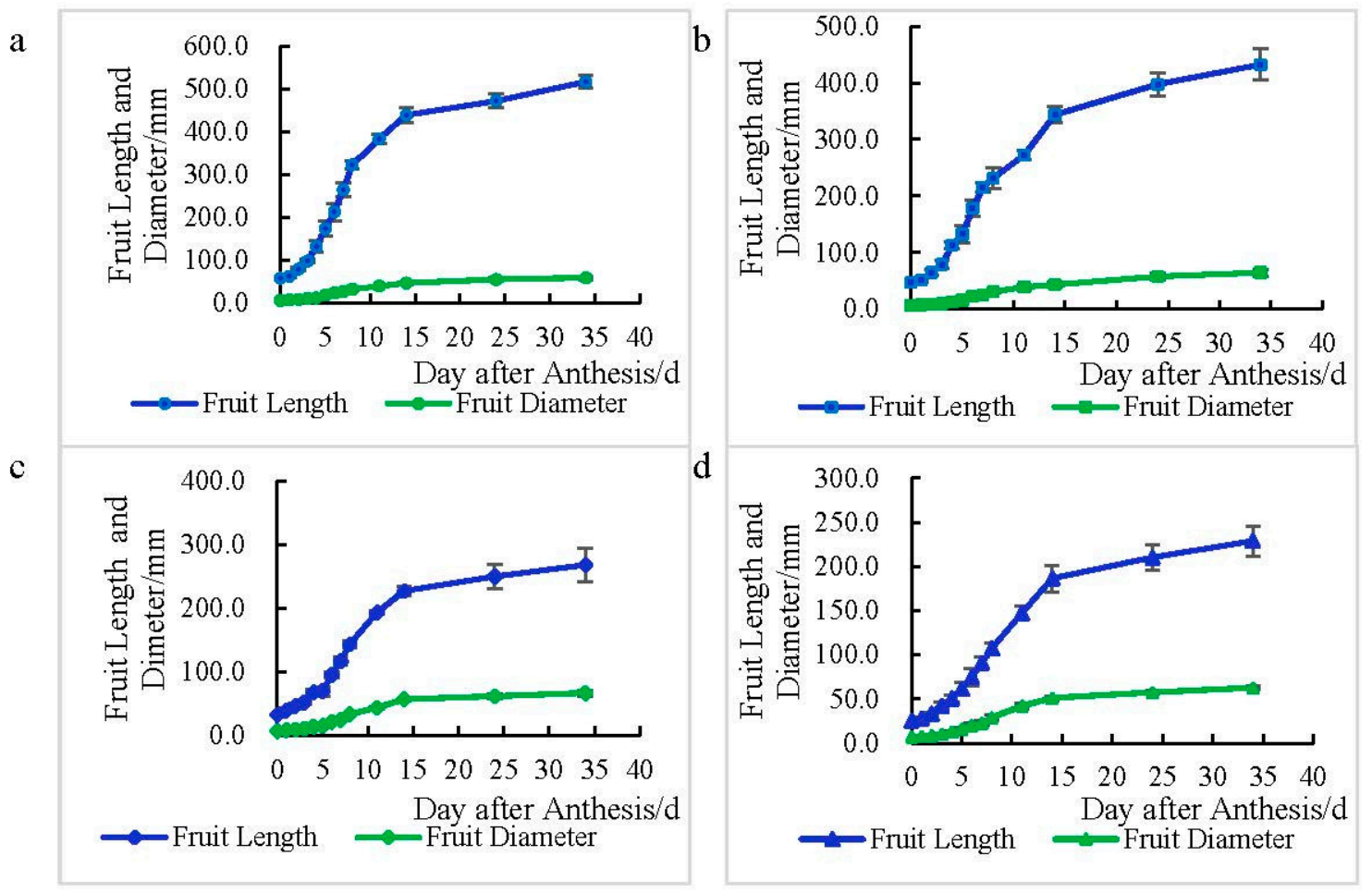

Figure 3. The dynamic growth of fruit length and fruit diameter in four types of cucumber fruits during the development of long fruit (a), medium long fruit (b), medium fruit (c) and short fruit (d).

As for the daily increment in fruit growth, three periods of rapid growth could be found in the development of both FL and FD in four types of bar fruits. For FL, the first period appeared at 3-5 DAA, followed by the peak at 8 DAA, and the last one came at 12-14 DAA. For FD, three growth peaks came in the similar period as FL, except the first period at 5-7 DAA, meaning that the development trend of FD was the same as for FL, but later in the first period. From the comparative analysis among different kinds of cucumber fruit, it was suggested that, except for increment, the growth trend of FL and FD did not show obvious difference among different kinds of cucumber fruits (Figure 4a,b), meaning that the development trend of FL and FD was hardly influenced by the shape and size of the cucumber fruit. From correlation analysis of developing FL and FD, the positive correlation of two indexes was showed in the whole development stage. Pearson correlation between dynamic developing FL and FD of long fruits, medium long fruits, medium fruits and short fruits was $0.970^{* *}, 0.970 * *, 0.975^{* *}$ and $0.936^{* *}$, respectively (** correlation is significant at the 0.01 level). As shown (Figure $5 \mathrm{a}-\mathrm{d}$ ), for the four types, the regression equations between $\mathrm{FL}(\mathrm{y})$ and $\mathrm{FD}(\mathrm{x})$ in turn were: $y=8.8261 x+13.487\left(\mathrm{R}^{2}=0.9911\right)$, $y=6.713 x+20.818\left(R^{2}=0.982\right), y=3.8978 x+11.672\left(R^{2}=0.9964\right)$ and $y=3.5715 x+4.3119$ $\left(R^{2}=0.9981\right)$, respectively. These results suggest the developments of FL and FD influence each other, but the specific influence mechanism is unclear. 


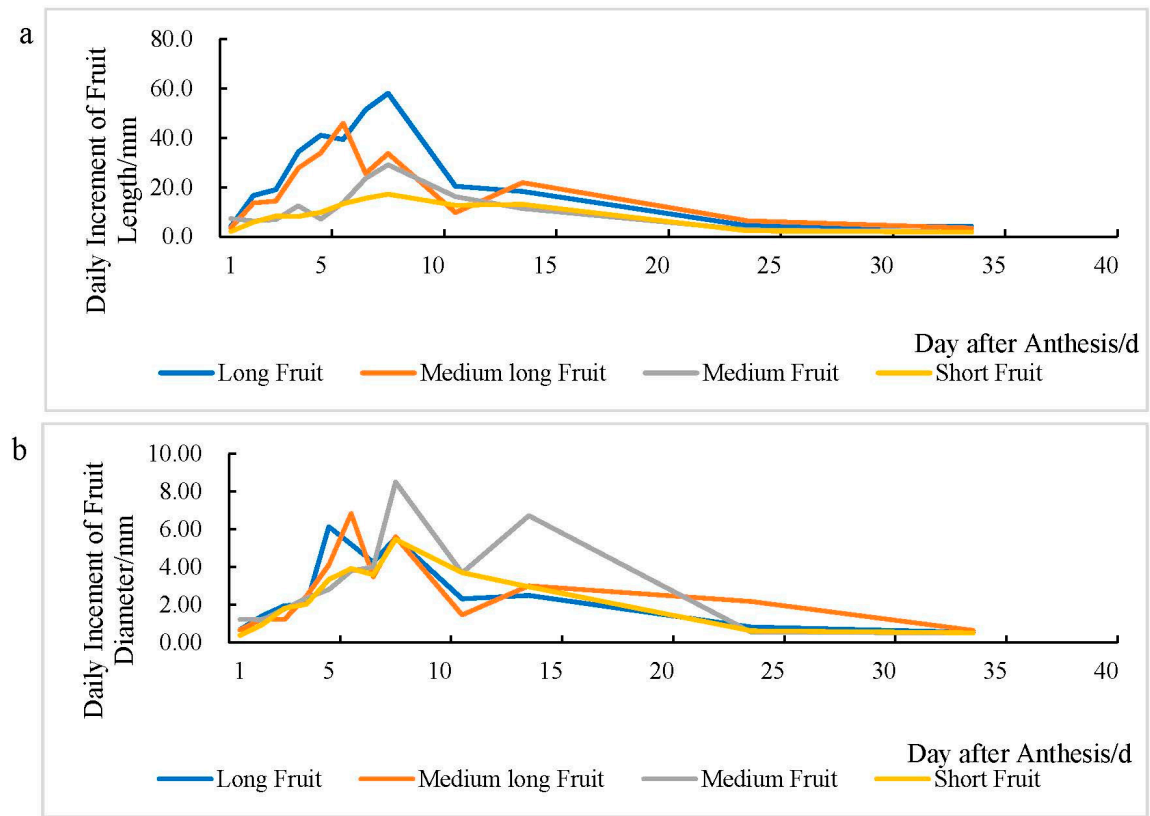

Figure 4. Daily growth of fruit length (a) and fruit diameter (b) in four types of cucumber fruits during fruit development process.
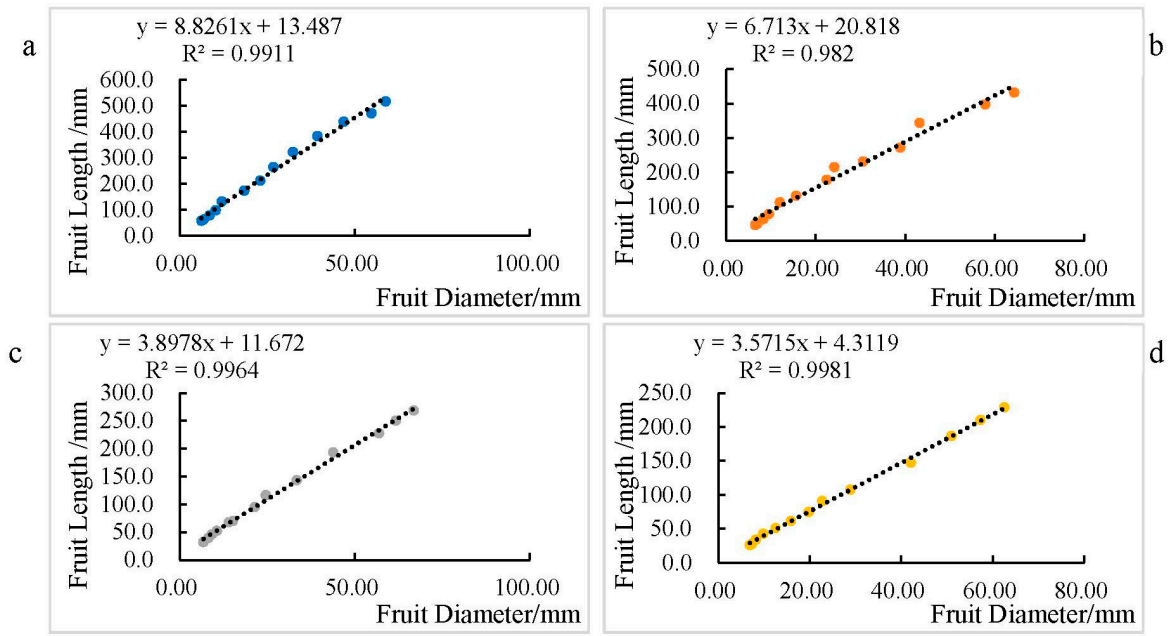

Figure 5. The correlations between fruit length and diameter of long fruit (a), medium long fruit (b), medium fruit (c) and short fruit (d).

For round fruit ("16-1"), the FL and FD also developed in a model "S" curve: Slow (before 5 DAA) -fast (5-15 DAA)-slow (after 15 DAA) (Figure S2). The development of FL and FD also showed significantly positive relation, as the Pearson correlation between dynamic developing FL and FD was $0.9922^{* *}(* *$ correlation is significant at the 0.01 level) and the regression equation between FL(y) and FD (x) was: $y=1.4234 x+10.459\left(r^{2}=0.9832\right)$ (Figure S3). However, compared to bar fruit, the development trend of FL and FD in round fruit always kept same and synchronous from ovary to mature fruit, and the fast development stage of both FL and FD in round fruit occurred later (5 DAA) than bar fruit (3 DAA). 


\subsection{Differences in Shape Traits of Other Organs of Different Types of Cucumber Fruits and Their Correlations to FSI}

The length, width and shape index of seed, cotyledon, true leaf, female flower petal and ovary were comparatively analyzed among four types of cucumber bar fruits and some differences and correlations to FSI were found. In addition, these shape traits were also investigated in round fruit (cucumber "16-1") and the correlations between the shape traits of different organs were analyzed.

\subsubsection{Shape Traits of Other Organs in Bar Fruit}

In comparison to seed shape traits, little difference was found among four types of bar fruits (Table 2). There was no significant difference in seed length and seed shape indexes among four types of cucumber fruits, but the seed width of medium fruits and short fruits was significantly larger $(p<0.05)$ than that of the medium long fruits.

Table 2. Seed shape traits of four types of bar fruits.

\begin{tabular}{cccc}
\hline Fruit Type & Length $(\mathbf{m m})$ & Width $(\mathbf{m m})$ & Seed Shape Index \\
\hline Long fruit & $10.25 \pm 0.30 \mathrm{a}$ & $3.91 \pm 0.05 \mathrm{ab}$ & $2.63 \pm 0.08 \mathrm{a}$ \\
Medium long fruit & $9.84 \pm 0.14 \mathrm{a}$ & $3.76 \pm 0.04 \mathrm{~b}$ & $2.63 \pm 0.04 \mathrm{a}$ \\
Medium fruit & $10.12 \pm 0.11 \mathrm{a}$ & $3.99 \pm 0.05 \mathrm{a}$ & $2.55 \pm 0.03 \mathrm{a}$ \\
Short fruit & $9.95 \pm 0.13 \mathrm{a}$ & $4.01 \pm 0.04 \mathrm{a}$ & $2.48 \pm 0.03 \mathrm{a}$ \\
\hline
\end{tabular}

Different letters indicate significant differences between means within columns of four types of cucumber fruits at $p<0.05$ by Tukey HSD test.

In comparison with cotyledon shape traits, significant differences were found in the length and the width of cotyledon, but no significant difference was found in the cotyledon shape index among different types of cucumber fruits (Table 3). As for cotyledon length, long fruits appeared the longest and significantly longer $(p<0.05)$ than that of the other three types of cucumber fruits, and there was no significant difference among the other three types of cucumber fruits. Generally, the cotyledon of longer fruits appeared wider than that of shorter fruits. The cotyledon width of the long fruits and the medium long fruits was significantly wider than that of the medium fruits and the short fruits. However, there was no significant difference between the long fruits and the medium long fruits, and the medium fruits and short fruits also did not show obvious difference.

Table 3. Cotyledon shape traits of four types of bar fruits.

\begin{tabular}{cccc}
\hline Fruit Type & Length $(\mathbf{m m})$ & Width $(\mathbf{m m})$ & Cotyledon Shape Index \\
\hline Long fruit & $40.7 \pm 0.9 \mathrm{a}$ & $22.67 \pm 0.38 \mathrm{a}$ & $1.80 \pm 0.03 \mathrm{a}$ \\
Medium long fruit & $38.1 \pm 0.9 \mathrm{ab}$ & $22.31 \pm 0.49 \mathrm{a}$ & $1.73 \pm 0.04 \mathrm{a}$ \\
Medium fruit & $35.1 \pm 0.8 \mathrm{~b}$ & $20.20 \pm 0.41 \mathrm{~b}$ & $1.74 \pm 0.02 \mathrm{a}$ \\
Short fruit & $35.0 \pm 0.9 \mathrm{~b}$ & $19.89 \pm 0.62 \mathrm{~b}$ & $1.78 \pm 0.04 \mathrm{a}$
\end{tabular}

Different letters indicate significant differences between means within columns of four types of cucumber fruits at $p<0.05$ by Tukey HSD test.

As for true leaf shape index, there was no significant difference among four types of cucumber fruits, but the long fruits and the true leaf of medium fruits were longer and wider than those of the medium long fruits and the short fruits (Table 4). 
Table 4. True leaf shape traits of four types of cucumber fruits.

\begin{tabular}{cccc}
\hline Fruit Type & Leaf Blade Length $\mathbf{( m m )}$ & Leaf Blade Width $(\mathbf{m m})$ & Leaf Shape Index \\
\hline Long fruit & $52.3 \pm 1.3 \mathrm{a}$ & $60.60 \pm 1.41 \mathrm{a}$ & $0.87 \pm 0.02 \mathrm{a}$ \\
Medium long fruit & $45.0 \pm 1.7 \mathrm{~b}$ & $51.99 \pm 1.83 \mathrm{c}$ & $0.84 \pm 0.03 \mathrm{a}$ \\
Medium fruit & $50.4 \pm 0.8 \mathrm{a}$ & $57.46 \pm 1.18 \mathrm{ab}$ & $0.88 \pm 0.01 \mathrm{a}$ \\
Short fruit & $45.4 \pm 0.9 \mathrm{~b}$ & $54.71 \pm 1.10 \mathrm{bc}$ & $0.83 \pm 0.01 \mathrm{a}$ \\
\hline
\end{tabular}

Different letters indicate significant differences between means within columns of four types of cucumber fruits at $p<0.05$ by Tukey HSD test.

As for female flower, longer fruits showed wider petal but smaller petal shape index generally (Table 5). While the petal width of the long fruits ranked the first and the short fruits ranked the last, the petal shape index appeared inverse. The petal shape indexes of short fruits and medium fruits were significantly higher than those of the long fruits.

Table 5. The female flower petal shape traits of four types of cucumber fruits.

\begin{tabular}{cccc}
\hline Fruit Type & Petal Length $\mathbf{( m m )}$ & Petal Width $(\mathbf{m m})$ & Petal Shape Index \\
\hline Long fruit & $21.93 \pm 0.32 \mathrm{ab}$ & $16.28 \pm 0.24 \mathrm{a}$ & $1.35 \pm 0.02 \mathrm{~b}$ \\
Medium long fruit & $23.04 \pm 0.43 \mathrm{a}$ & $15.03 \pm 0.26 \mathrm{a}$ & $1.56 \pm 0.05 \mathrm{ab}$ \\
Medium fruit & $19.54 \pm 0.58 \mathrm{c}$ & $12.81 \pm 0.41 \mathrm{~b}$ & $1.56 \pm 0.06 \mathrm{a}$ \\
Short fruit & $21.15 \pm 0.50 \mathrm{bc}$ & $12.59 \pm 0.48 \mathrm{~b}$ & $1.76 \pm 0.09 \mathrm{a}$ \\
\hline
\end{tabular}

Different letters indicate significant differences between means within columns of four types of cucumber fruits at $p<0.05$ by Tukey HSD test.

From the comparative analysis of ovary traits among different types of cucumber fruits, it was found that the longer of the ovary length and the lager of the ovary shape index were, the longer the type of cucumber fruit would be. Significant differences were found among all four types of fruits in ovary length and ovary shape index, while significant difference only existed between long fruits and short fruits (Table 6). As for carpopodium, there showed some differences in length, width and shape index. In general, longer cucumber fruits showed a little smaller carpopodium shape index.

Table 6. The shape traits of ovary and its carpopodium of four types of cucumber fruits.

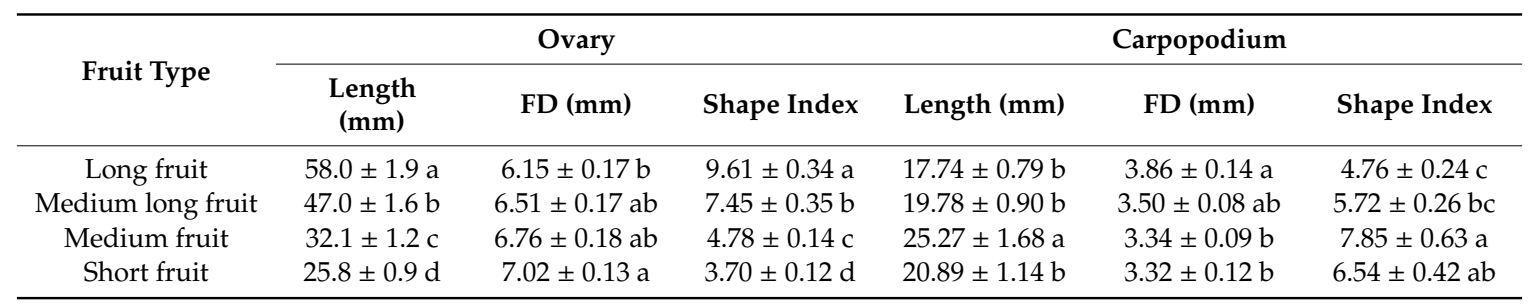

Different letters indicate significant differences between means within columns of four types of cucumber fruits at $p<0.05$ by Tukey HSD test.

\subsubsection{Correlations of Fruit Shape to Other Organ Shapes in Bar Fruit}

The correlations of FSI in both commodity fruit stage and mature fruit stage to shape traits of other organs were analyzed among different types of cucumber fruits (Table S1). The results showed that, for shape index, there existed significant positive correlation among ovary, commodity fruit and mature fruit, and negative correlation between fruit (both commodity fruit and mature fruit) and ovary carpopodium, female flower petal and pedicel. For seed, cotyledon and true leaf, no correlation was found between the shape index of them and that of fruit (neither commodity fruit nor mature fruit).

For FL and FD in commodity fruit, the correlations to the length and width of other organs were also investigated (Table S2). The result exhibited that there existed correlation between many traits and FL, among which the length and width of cotyledon, the length and width of true leaf, the length 
of true leaf petiole, the length and width of female flower petal and the length of ovary were positively correlated, while the width of seed, the width of ovary, the length of ovary carpopodium and the length of pedicel were negatively correlated. For FD, smaller correlations were found concerning length and width of other organs; however, length and width of seed, width of ovary, length of ovary carpopodium and length of pedicel were positively correlated and length of ovary was negatively correlated.

\subsubsection{Shape Traits of Different Organs and Correlation Analysis in Round Fruit (Cucumber "16-1")}

For cucumber accession "16-1", its fruit always showed less FSI with less FL and larger FD from ovary to mature fruit (Table 7 and Table S3). Similarly, the shape index of seed was also smaller than that of bar fruit. In addition to these different appearance from bar fruit, the shape index of cotyledon of cucumber "16-1" was observed to be larger, because of the smaller length.

Table 7. Shape indexes of different organs in cucumber "16-1".

\begin{tabular}{ccccccccc}
\hline Organ & Seed & Cotyledon & True Leaf & Petal & Ovary & Carpopodium & $\begin{array}{c}\text { Commodity } \\
\text { Fruit }\end{array}$ & $\begin{array}{c}\text { Mature } \\
\text { Fruit }\end{array}$ \\
\hline $\begin{array}{c}\text { Shape } \\
\text { index }\end{array}$ & $2.16 \pm 0.03$ & $3.39 \pm 0.40$ & $0.94 \pm 0.04$ & $1.74 \pm 0.09$ & $2.22 \pm 0.02$ & $5.35 \pm 0.12$ & $1.73 \pm 0.03$ & $1.46 \pm 0.02$ \\
\hline
\end{tabular}

Correlation of shape indexes among these organs in round fruit "16-1" and long fruit type was analyzed (Table 8 ). As a result, significantly positive correlation was found between the shape index of fruits and that of seed as well as ovary, and significantly negative correlation was shown in the shape indexes between fruit and cotyledon as well as true leaf.

Table 8. Pearson Correlation among shape indexes of different organs.

\begin{tabular}{ccccccc}
\hline Organ & Seed & Cotyledon & True Leaf & Petal & Ovary & Carpopodium \\
\hline $\begin{array}{c}\text { Pearson } \\
\text { Correlation }\end{array}$ & $0.799^{* *}$ & $-0.740^{* *}$ & $-0.588^{*}$ & $-0.791^{* *}$ & $0.911^{* *}$ & 0.432 \\
\hline
\end{tabular}

* Correlation is significant at the 0.05 level; ${ }^{* *}$ correlation is significant at the 0.01 level.

\section{Discussion}

In this work, we employed 27 accessions with bar fruit and one accession with round fruit and 27 accessions with bar fruit were classified into four types. Previous work has conducted many classifications and evaluations of different cucumber accessions based on commercial traits of fruit (weight and size) [17], traits associated to yield (seed germination and flowering time) $[18,19]$ and genetic variability, heritability as well as genetic advance [20]. In this work, we classified 27 different inbred lines into four types based on the morphological indexes (shape and size) of different organs, like seed, flower, cotyledon, true leaf, fruit and so on, without involving genome or origin as factors, which might cause some difference from previous work.

Results in this work showed that in both the four types of bar fruits and round fruits, the development of FL and FD were both shown in the model of an "S" curve: Slow (before 5 DAA) -fast (5-15 DAA)-slow (after 15 DAA). This growth pattern is consistent with the results of some previous studies, with little variation due to the cultivar and growth environment $[14,21-24]$. The similar development trend suggests the development pattern of FL and FD is little influenced by the fruit shape and size.

For crops harvested at an early stage, the fruit growth process can be divided into three stages: Ovary development stage, cell division stage and cell expansion stage [25]. The number and size of cells determine the final size of the fruit. On regulating the development of FL and FD, previous studies indicated that it attributed to the various rate and timing of cell division and expansion. The number of cells in cucumber fruits increased rapidly at 0-6 DAA and then slowed down. In most cases of cultivated cucumber, rapid cell division occurs before anthesis and continues for 0-5 DAA, 
followed by a sharp increase in cell size at $4-12$ DAA; then the cell expansion is largely completed at 12-16 DAA $[25,26]$. This development of cells may account for the growth pattern of FL and FD.

In this study, the FSI of four types of bar fruits all changed in a "Double-S" pattern, with two obvious increases during 0-4 DAA and 6-7 DAA. Combined with the development of FL and FD, it can be deduced that fruit elongation developed earlier than radial growth at first, then FD speeded up to follow FL. After another alternately rapid growth period of FL and FD, the fruit developed synchronously after 14 DAA. Compared to bar fruit, the dynamic change curve of FL and FD in round fruit always showed the same trend, suggesting FL and FD always keep synchronous growing in the whole development process.

In the whole growth process, high positive correlation was confirmed between the development of FL and that of FD in both bar fruits and round fruits, indicating the growth of FL and FD influence each other and are little influenced by the shape and size of the cucumber. However, there were exhibited differences in the time and speed of fruit elongation and radial growth, which may be caused by the change of planes of cell division and isotropic and anisotropic cell enlargement during development [21,27].

About the correlation among organ development, high positive correlation among FSI of ovary, commodity fruit and mature fruit suggested that the fruit shape is controlled by critical factors before anthesis and little change occurs during development [21,27-33]. For all FL, FD and FSI, high positive correlation existed among ovary, commodity fruit and mature fruit, meaning the shape is decided in the ovary period and will not change a lot, indicating ovary traits (FL, FD and FSI) can be used to predict shape and size of a cucumber fruit preliminarily. Besides, what is interesting is that both the length and width of cotyledon, true leaf and female flower petal are positively related to the FL, meaning that the plants with larger cotyledons, true leaves or female flower petals have more potential to produce longer fruits. For FD, the length and width of seed, length of ovary carpopodium and length of carpopodium were positively correlated, suggesting plants from larger seed may produce thicker fruits and longer fruits. When the carpopodium is longer, the FD may be bigger. These results were similar with the correlations among different organs and the size of strawberry fruit [13], which increases the reliability of the correlation among different organs. For seed, cotyledon and true leaf, no correlation was found between the shape index of them and fruit. However, when these organs' traits were compared to "16-1" and long fruit type, significant correlations were found between the shape index of fruits and that of seed, cotyledon and true leaf. This different result may be due to the character of specific accession or the big difference in shape and size, which needs further study.

The correlation between different organs can be helpful for breeders to predict the shape and size of cucumber fruit from the shape traits of seed, cotyledon, leaf and flower, so the breeding process can be shortened. For farmers, they can observe the shape and size of other organs to predict relative traits of fruit, and based on the prediction can estimate the development status of fruit, so they can harvest commodity fruit or adjust cultivation management in time.

In production, the fluctuating change of FSI indicates the FL and FD develop alternately before $8 \mathrm{DAA}$, a key period for the formation of good appearance quality. Due to the important influence of environment on fruit development, more attention should be paid to careful cultivation management at this stage, especially ensuring enough irrigation and fertilization in time. After 14 DAA, the FSI became stable, which means the FL and FD began to simultaneously grow. While the FL and FD keep continuously developing at a slow speed after the commodity maturity (11-14 DAA), the developing fruits continuously abstract nutrition from plants [34]. However, after the commodity stage, the edible value of the fruits starts to decrease, with yellow skin and hard flesh, so it is necessary to harvest in time, not only for the good quality of commodity fruit but also for the proper distribution of nutrition to potential fruits, which is important for high quality and abundant yield. 


\section{Conclusions}

Our results indicated that the development trend of FL and FD in cucumbers were little affected by the shape and size of a fruit, showing an "S" curve: Slow (before 5 DAA)-fast (5-15 DAA)-slow (after 15 DAA). The development of FSI in a "Double-S" curve suggested that the elongation growth of cucumber fruit developed earlier than the radial growth at first, then the FL and FD developed alternatively. In the whole development, high positive correlation existed between FL and FD, meaning the developments of FL and FD have an effect on each other.

For correlations to the size of different organs, high positive correlation existed among FSI of ovary, commodity fruit and mature fruit, and negative correlation existed between FSI (both commodity fruit and mature fruit) and ovary carpopodium shape index, female flower petal shape index and pedicel shape index. For FL in commodity fruit, both the length and width of cotyledon, true leaf and female flower petal are positively related, while width of seed, width of ovary, length of ovary carpopodium and length of pedicel are negatively correlated. For FD in commodity fruit, smaller correlations were found with length and width of other organs; however, length and width of seed, width of ovary, length of ovary carpopodium and length of pedicel were positively correlated and length of ovary was negatively correlated.

Supplementary Materials: The following are available online at http://www.mdpi.com/2073-4395/10/8/1111/s1, Figure S1: Dynamic changes of fruit shape index in a round shape fruit cucumber "16-1". Figure S2: Dynamic growth of fruit length and fruit diameter in a round shape fruit cucumber "16-1" during fruit development. Figure S3: Correlations between fruit length and diameter of a round shape fruit cucumber "16-1". Table S1: Pearson correlations of FSI in both commodity fruit and mature fruit to shape index of other organs. Table S2: Pearson correlations of FL and FD to the length and width of other organs. Table S3: The shape traits of different organs in cucumber "16-1". Table S4: Cucumber inbred lines used in this study and the basic information.

Author Contributions: M.C. analyzed the data and wrote the manuscript. Y.Y. conducted the experiment and collected data. Z.C. and H.M. designed and supervised the study. All authors have read and agreed to the published version of the manuscript.

Funding: This research was supported by National Key R\&D Program of China (2016YFD0101705-6).

Conflicts of Interest: The authors declare no conflict of interest.

\section{References}

1. Qi, J.; Liu, X.; Shen, D.; Miao, H.; Xie, B.; Li, X.; Zeng, P.; Wang, S.; Shang, Y.; Gu, X.; et al. A genomic variation map provides insights into the genetic basis of cucumber domestication and diversity. Nat. Genet. 2013, 45, 1510-1515. [CrossRef] [PubMed]

2. Pan, Y.; Liang, X.; Gao, M.; Liu, H.; Meng, H.; Weng, Y.; Cheng, Z. Round fruit shape in WI7239 cucumber is controlled by two interacting quantitative trait loci with one putatively encoding a tomato SUN homolog. Theor. Appl. Genet. 2016, 130, 573-586. [CrossRef] [PubMed]

3. Weng, Y.; Colle, M.; Wang, Y.; Yang, L.; Rubinstein, M.; Sherman, A.; Ophir, R.; Grumet, R. QTL mapping in multiple populations and development stages reveals dynamic quantitative trait loci for fruit size in cucumbers of different market classes. Theor. Appl. Genet. 2015, 128, 1747-1763. [CrossRef] [PubMed]

4. Pan, Y.; Wang, Y.; McGregor, C.; Liu, S.; Luan, F.; Gao, M.; Weng, Y. Genetic architecture of fruit size and shape variation in cucurbits: A comparative perspective. Theor. Appl. Genet. 2019, 133, 1-21. [CrossRef]

5. Gillaspy, G.; Ben-David, H.; Gruissem, W. Fruits: A developmental perspective. Plant Cell 1993, 5, 1439-1451. [CrossRef]

6. Okello, R.C.O.; Heuvelink, E.; De Visser, P.H.B.; Struik, P.C.; Marcelis, L.F. What drives fruit growth? Funct. Plant Boil. 2015, 42, 817-827. [CrossRef]

7. Pan, Y.; Meng, H.; Cheng, S. Research progress on fruit shape of vegetable crops. China Veg. 2013, 4, 6-13.

8. Qiao, J.; Liu, F.; Chen, Y.; Lian, Y. Research progress on inheritance of fruit shape in horticultural crops. Acta Hortic. Sin. 2011, 38, 1385-1396.

9. Tan, J.; Tao, Q.; Niu, H.; Zhang, Z.; Li, D.; Gong, Z.; Weng, Y.; Li, Z. A novel allele of monoecious (m) locus is responsible for elongated fruit shape and perfect flowers in cucumber (Cucumis sativus L.). Theor. Appl. Genet. 2015, 128, 2483-2493. [CrossRef] 
10. Pan, D. Research on rule of cucumber fruit growth and cell division. J. Northeast Agric. Univ. 2010, 3, 33-37.

11. Ando, K.; Grumet, R. Transcriptional Profiling of Rapidly Growing Cucumber Fruit by 454-Pyrosequencing Analysis. J. Am. Soc. Hortic. Sci. 2010, 135, 291-302. [CrossRef]

12. Ding, X.; Jiang, Y.; Zhang, Z.; Jin, H.; Zhang, H.; Yu, J. The change of cucumber growth and development in glasshouse. Acta Agric. Shanghai 2013, 29, 36-39.

13. Jiang, Y.; Zhang, J.; Zhang, X. Studies on the growth and development of Cucumis sativus fruit and the relations between vertical diameter and cross section and weight of Cucumis Sativus fruits. Acta Agric. Univ. Henanensis 1999, 33, 123-124, 136.

14. Yang, X.Y.; Wang, Y.; Jiang, W.J.; Liu, X.L.; Zhang, X.M.; Yu, H.J.; Huang, S.W.; Liu, G.Q. Characterization and expression profiling of cucumber kinesin genes during early fruit development: Revealing the roles of kinesins in exponential cell production and enlargement in cucumber fruit. J. Exp. Bot. 2013, 64, 4541-4557. [CrossRef] [PubMed]

15. Wang, X.S.; Li, B.X.; Huang, J.L. Studies on the correlation between plant morphologh and its fruit in strawberry. Acta Agric. Boreali-Occident. Sin. 1997, 6, 74-77.

16. Li, H. Study on the Phylogenetic Relationship and Characteristics among Chinese Cucumber (Cucumis Sativus L.) Commercial Variety. Master's Thesis, Northwest A\&F University, Xianyang, China, 2008.

17. Valcárcel, J.V.; Peiró, R.M.; Pérez-de-Castro, A.; Díez, M.J. Morphological characterization of the cucumber (Cucumis sativus L.) collection of the COMAV's Genebank. Genet. Resour. Crop. Evol. 2018, 65, 1293-1306. [CrossRef]

18. Khan, Z.; Shah, A.; Gul, R.; Majid, A.; Khan, U.; Ahmad, H. Morpho-agronomic characterization of cucumber germplasm for yield and yield associated traits. Int. J. Agron. Agric. Res. 2016, 6, 1-6.

19. Golabadi, M.; Golkar, P.; Eghtedary, A.-R. Assessment of genetic variation in cucumber (Cucumis sativus L.) genotypes. Eur. J. Exp. Biol. 2012, 2, 1382-1388.

20. Gaikwad, A.; Musmade, A.; Dhumal, S.; Sonawane, H. Variability studies in cucumber (Cucumis sativus L.). Ecol. Environ. Conserv. 2011, 17, 799-802.

21. Colle, M.; Weng, Y.; Kang, Y.; Ophir, R.; Sherman, A.; Grumet, R. Variation in cucumber (Cucumis sativus L.) fruit size and shape results from multiple components acting pre-anthesis and post-pollination. Planta 2017, 246, 641-658. [CrossRef]

22. Fu, F.Q.; Mao, W.H.; Shi, K.; Zhou, Y.H.; Asami, T.; Yu, J.-Q. A role of brassinosteroids in early fruit development in cucumber. J. Exp. Bot. 2008, 59, 2299-2308. [CrossRef] [PubMed]

23. Fu, F.Q.; Mao, W.H.; Shi, K.; Zhou, Y.H.; Yu, J. Spatio-temporal changes in cell division, endoreduplication and expression of cell cycle-related genes in pollinated and plant growth substances-treated ovaries of cucumber. Plant Boil. 2010, 12, 98-107. [CrossRef] [PubMed]

24. Marcelis, L.F.M.; Hofman-Eijer, L.R.B. Cell division and expansion in the cucumber fruit. J. Hortic. Sci. 1993, 68, 665-671. [CrossRef]

25. Grumet, R.; Colle, M. Genomic Analysis of Cucurbit Fruit Growth. In Comparative and Evolutionary Genomics of Angiosperm Trees; Springer Science and Business Media: Berlin, Germany, 2016; Volume 20, pp. 321-344.

26. Hormone, E. Effect of pollination on cell division, cell enlargement, and endogenous hormones in fruit development in a gynoecious cucumber. Sci. Hortic. 2008, 116, 1-7.

27. Liu, X.X.; Pan, Y.P.; Liu, C.; Ding, Y.Y.; Wang, X.; Cheng, Z.H.; Meng, H.W. Cucumber fruit size and shape variations explored from the aspects of morphology, histology, and endogenous hormones. Plants 2020, 9, 772. [CrossRef]

28. Wu, S.; Zhang, B.; Keyhaninejad, Y. Tomato reveals a common mechanism underlying morphological diversification in domesticated plants. Nat. Commun. 2018, 9, 4734. [CrossRef]

29. Bo, K.; Ma, Z.; Chen, J.; Weng, Y. Molecular mapping reveals structural rearrangements and quantitative trait loci underlying traits with local adaptation in semi-wild Xishuangbanna cucumber (Cucumis sativus L. var. xishuangbannanesis Qi et Yuan). Theor. Appl. Genet. 2014, 128, 25-39. [CrossRef]

30. Pan, Y.; Qu, S.; Bo, K.; Gao, M.; Haider, K.R.; Weng, Y. QTL mapping of domestication and diversifying selection related traits in round-fruited semi-wild Xishuangbanna cucumber (Cucumis sativus L. var. xishuangbannanesis). Theor. Appl. Genet. 2017, 130, 1531-1548. [CrossRef]

31. Shimomura, K.; Fukino, N.; Sugiyama, M.; Kawazu, Y.; Sakata, Y.; Yoshioka, Y. Quantitative trait locus analysis of cucumber fruit morphological traits based on image analysis. Euphytica 2017, 213, 213. [CrossRef] 
32. Sinnott, E.W. A Developmental Analysis of Inherited Shape Differences in Cucurbit Fruits. Am. Nat. 1936, 70, 245-254. [CrossRef]

33. Wei, Q.-Z.; Fu, W.-Y.; Wang, Y.-Z.; Qin, X.-D.; Wang, J.; Li, J.; Lou, Q.; Chen, J. Rapid identification of fruit length loci in cucumber (Cucumis sativus L.) using next-generation sequencing (NGS)-based QTL analysis. Sci. Rep. 2016, 6, 27496. [CrossRef] [PubMed]

34. Chen, C.; Xiang, B. Growth and development properties of cucumber under modern greenhouse. Trans. CSAE 2005, 21, 189-193. article distributed under the terms and conditions of the Creative Commons Attribution (CC BY) license (http://creativecommons.org/licenses/by/4.0/). 